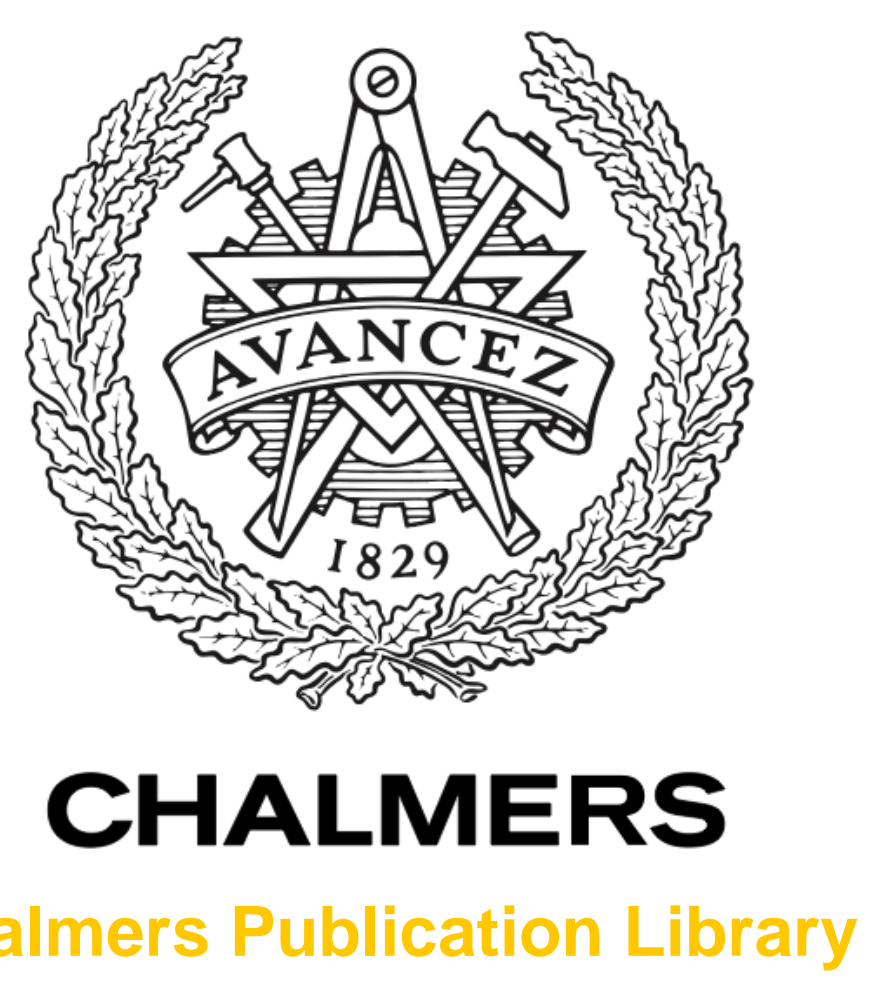

Chalmers Publication Library

\title{
An 802.11p Cross-Layered Pilot Scheme for Time- and Frequency-Varying Channels and Its Hardware Implementation
}

This document has been downloaded from Chalmers Publication Library (CPL). It is the author's version of a work that was accepted for publication in:

IEEE Transactions on Vehicular Technology (ISSN: 0018-9545)

Citation for the published paper:

Nagalapur, K. ; Brännström, F. ; Ström, E. et al. (2016) "An 802.11p Cross-Layered Pilot Scheme for Time- and Frequency-Varying Channels and Its Hardware Implementation".

IEEE Transactions on Vehicular Technology, vol. 65(6), pp. 3917-3928.

http://dx.doi.org/10.1109/TVT.2016.2550661

Downloaded from: http://publications.lib.chalmers.se/publication/239399

Notice: Changes introduced as a result of publishing processes such as copy-editing and formatting may not be reflected in this document. For a definitive version of this work, please refer to the published source. Please note that access to the published version might require a subscription.

Chalmers Publication Library (CPL) offers the possibility of retrieving research publications produced at Chalmers University of Technology. It covers all types of publications: articles, dissertations, licentiate theses, masters theses, conference papers, reports etc. Since 2006 it is the official tool for Chalmers official publication statistics. To ensure that Chalmers research results are disseminated as widely as possible, an Open Access Policy has been adopted.

The CPL service is administrated and maintained by Chalmers Library. 


\title{
An 802.11p Cross-Layered Pilot Scheme for Time- and Frequency-Varying Channels and Its Hardware Implementation
}

\author{
Keerthi Kumar Nagalapur, Fredrik Brännström, Member, IEEE, Erik G. Ström, Senior Member, IEEE, \\ Fabian Undi, and Kim Mahler
}

\begin{abstract}
Robust channel estimation in IEEE 802.11p systems in highly time- and frequency-varying vehicular channels in combination with long data packets is a challenging task due to the ill-suited pilot pattern. Solutions of increased receiver complexity that use decision feedback and iterative decoding have been proposed to overcome the difficulty in robust channel estimation. In this work, a cross-layered method to introduce complementary training symbols into an $\mathbf{8 0 2 . 1 1 p}$ frame is proposed. In the proposed approach, known bits are multiplexed with the data in higher layers and a modified receiver can utilize these bits as training data for improved channel estimation. A standard receiver treats these bits as data and passes them to the higher layers where they can be removed, making the method compatible with the standard 802.11p transceivers. A software/firmware update of the higher layers is needed in a standard receiver to remove the multiplexed bits. A modified receiver with low complexity channel estimation schemes that utilizes the complementary training symbols is implemented in a field programmable gate array platform. Frame error rate measurements have been performed by interfacing the hardware implementation with a channel emulator. The measurement results follow the computer simulation results validating the hardware implementation. Moreover, measurement results show that the modified receiver follows the performance of an ideal receiver that has full knowledge of the channel (with only an offset in signal-to-noise ratio) and significantly outperforms the commercial 802.11p transceiver we tested.
\end{abstract}

Index Terms-IEEE 802.11p, cross layer, pilot scheme, OFDM, channel estimation, FPGA implementation.

\section{INTRODUCTION}

Vehicular communications, broadly classified into vehicleto-vehicle (V2V) and vehicle-to-infrastructure (V2I) communications, are expected to be a part of the future transportation systems. Vehicular communications will enable a plethora of applications ranging from traffic safety, traffic efficiency,

Copyright (C) 2015 IEEE. Personal use of this material is permitted. However, permission to use this material for any other purposes must be obtained from the IEEE by sending a request to pubs-permissions@ieee.org.

Keerthi Kumar Nagalapur, Fredrik Brännström, and Erik G. Ström are with the Division of Communication Systems, Department of Signals and Systems, Chalmers University of Technology, SE-412 96, Gothenburg, Sweden. E-mail: \{keerthi, fredrik.brannstrom, erik.strom\}@ chalmers.se. Fabian Undi and Kim Mahler are with the Department of Wireless Communications and Networks, Fraunhofer Heinrich Hertz Institute, Berlin, Germany. E-mail: \{fabian.undi, kim.mahler\}@hhi.fraunhofer.de

The research was funded by Chalmers Antenna Systems Excellence Center in the project 'Antenna Systems for V2X Communication'. Visits to Fraunhofer Heinrich Hertz Institute, Berlin, have been partially funded by COST IC 1004 STSM and Ericsson research foundation grants. and fuel efficiency. An extensive list of basic set of applications supported by vehicular communications under intelligent transportation systems (ITS) can be found in [1]. Designing a reliable wireless communication system necessitates a thorough understanding of the environments in which they will be deployed. Consequently, several vehicular channel measurement campaigns and characterization studies have been conducted. An extensive survey on vehicular channels, and a thorough discussion on the characteristics of vehicular channels and their impact on the design of reliable communication systems can be found in [2]. Delay and Doppler spreads of vehicular channels measured in the DRIVEWAY'09 campaign are analyzed using local scattering function in [3]. Coherence times as low as $180 \mu \mathrm{s}$ and coherence bandwidths as low as $200 \mathrm{kHz}$ are reported. The literature survey on vehicular channel measurements suggests that the vehicular channels are traffic scenario dependent and can be highly time- and frequency-selective.

IEEE $802.11 \mathrm{p}$ has been chosen as the physical (PHY) layer for vehicular communications [4], [5]. The pilot pattern in 802.11p [6], [7] is identical to the pattern in IEEE 802.11a. 802.11a was originally designed for relatively static indoor environments with pilots densely concentrated at the beginning of a frame. This combined with the highly time- and frequency-selective nature of vehicular channels makes robust channel estimation a challenging task. Several solutions have been proposed to address the problem of channel estimation in $802.11 \mathrm{p}$ systems. A channel estimation scheme called spectral temporal averaging (STA) is proposed in [8]. The scheme uses decisions from data subcarriers in combination with averaging in time and frequency domains to compute updated channel estimates. A channel estimation scheme called constructed data pilots (CDP) that uses preamble-based channel estimates to construct pilots from data symbols for further channel estimation is proposed in [9]. The STA and CDP schemes show improvement in frame error rate (FER) performance in comparison to the pilot-only based schemes. However, the performance has not been compared to the case with perfect channel state information (CSI). In [10], an early work on supporting higher velocities in 802.11a, a channel estimation scheme that tracks the channel impulse response by using the detected and remodulated data symbols, is presented. In [11], a receiver with iterative channel estimation making use of discrete prolate spheroidal sequences is proposed, and the receiver achieves FER performance close to the case 
with perfect CSI. A soft iterative receiver that uses factor graphs for performing joint channel estimation and detection is investigated in [12]. The receiver shows a significant FER performance improvement in comparison to the noniterative receivers. It is also reported in both [11] and [12] that the insertion of a postamble enables reduction of the number of iterations required to attain a fixed performance. The iterative schemes discussed above suggest that it is possible to perform robust channel estimation using the existing pilot pattern at the cost of increased receiver complexity. A scheme requiring modification in the $802.11 \mathrm{p}$ physical layer standard referred to as tentpoles scheme where a subset of data symbols is protected by a strong error control code is proposed in [13]. In this scheme, the data encoded with the strong code is decoded first and used as pilots for the channel estimation.

As an alternative to increasing the receiver complexity, pilot patterns suitable for low complexity channel estimation are proposed. In [14], another early work on supporting mobility in $802.11 \mathrm{a}$, frame formats consisting of modified pilot patterns called enhanced pilot (EP) patterns are proposed. One of the proposed pattern uses periodically inserted orthogonal frequency division multiplexing (OFDM) training symbols referred to as midambles. Channel estimation schemes to exploit the midambles are also described in the same paper. The concept of midambles was revisited in [15], where a channel tracking algorithm based on midambles is described. A modified frame format that consists of a single pilot per OFDM symbol, where the pilots in consecutive symbols are spaced two subcarriers apart, is proposed in [16]. These studies suggest that pilots spread throughout the entire frame allow robust channel estimation with low receiver complexity. However, these proposals require a modification to the $802.11 \mathrm{p}$ standard.

In our previous work [17], we proposed a cross-layered approach to periodically insert complementary pilot OFDM symbols into the $802.11 \mathrm{p}$ frame. In contrast to other works that introduce pilots by modifying the transmitter PHY layer, we propose a method to insert pilots by manipulating the transmitted data above the medium access control (MAC) layer. The extra pilots can be generated without any modification to the transmitter PHY or MAC layer. A modified receiver can utilize the inserted pilots to improve the channel estimation. For a standard receiver incapable of using the complementary pilots, these extra pilots are seen as part of data and are decoded using the normal procedure. As a result, a standard $802.11 \mathrm{p}$ receiver is compatible with our scheme. The standard receiver requires a simple software/firmware update in the higher layers to remove these additional pilots. The proposed complementary training pilots provide more support for channel estimation and are not specific to any particular channel estimation technique.

In this work, we have implemented a modified receiver that utilizes the complementary pilots in a field programmable gate array (FPGA) based hardware platform to show the implementation feasibility of our method. The modified receiver is implemented on High Performance Digital Radio Testbed (HIRATE), a general purpose FPGA based platform for rapid prototyping developed at Fraunhofer Heinrich Hertz Institute, Berlin [18]. The modified receiver is implemented

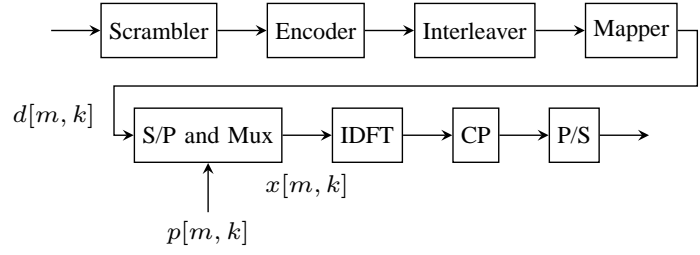

Fig. 1. Block diagram of an 802.11p transmitter.

by extending an existing HIRATE standard compliant $802.11 \mathrm{p}$ transceiver implementation. The main contributions of the paper are: (i) we present an improved version of the algorithm proposed in our previous work [17] to insert complementary training data before the PHY and MAC layers into an 802.11p frame; (ii) linear interpolation which can be easily implemented in hardware is used to utilize the inserted training data for channel estimation; (iii) a modified receiver that utilizes the complementary pilots has been implemented in an FPGA platform; (iv) experiments have been performed to validate the compatibility of the proposed scheme with the standard 802.11 transceivers and to measure the performance of the channel estimation based on linear interpolation.

The paper is organized as follows. Section II describes the system model and the $802.11 \mathrm{p}$ frame format. The proposed frame format and the method to introduce complementary training symbols are explained in Section III. In Section IV, receiver configuration for utilizing the complementary training symbols is described. Section V briefly explains the FPGA implementation of the proposed scheme. Measurement setup and FER performance are discussed in Section VI. Section VII concludes the paper.

\section{System Model and 802.11P Frame Structure}

The PHY layer of 802.11p uses OFDM with $N=64$ subcarriers and a cyclic prefix (CP) of length $N_{\mathrm{CP}}=16$. Among the 64 subcarriers, 48 are allocated for data, 4 are allocated for pilots, and 12 are null subcarriers. A channel spacing of $10 \mathrm{MHz}$ results in OFDM symbol duration of $T_{\mathrm{SYM}}=8 \mu \mathrm{s}$ which includes a $\mathrm{CP}$ of duration $T_{\mathrm{CP}}=1.6 \mu \mathrm{s}$. The $802.11 \mathrm{p}$ standard supports eight different modulation and coding schemes (MCSs) [6, Table 18.4].

The encoding process of an $802.11 \mathrm{p}$ OFDM symbol is illustrated in Fig. 1. Data bits are passed through a scrambler which is initialized with a pseudo-random nonzero seed for every transmitted frame. The scrambled bits are encoded using a rate $1 / 2,(171,133)_{8}$ convolutional code. Higher coding rates are achieved using puncturing. The encoded bits are divided into groups of $N_{\mathrm{CBPS}}$ bit, where $N_{\mathrm{CBPS}}$ is the number of coded bits per OFDM symbol. The bits in each group are interleaved and mapped to the constellation $\mathcal{C}$ resulting in complex valued data symbols. The complex valued data and pilot symbols are mapped to the data subcarriers and the pilot subcarriers of the OFDM symbol, respectively. Following which, an $N$-point inverse discrete Fourier transform (IDFT) is performed to obtain the time domain signal and a CP of $N_{\mathrm{CP}}$ samples is added to form the OFDM symbol. For specific 


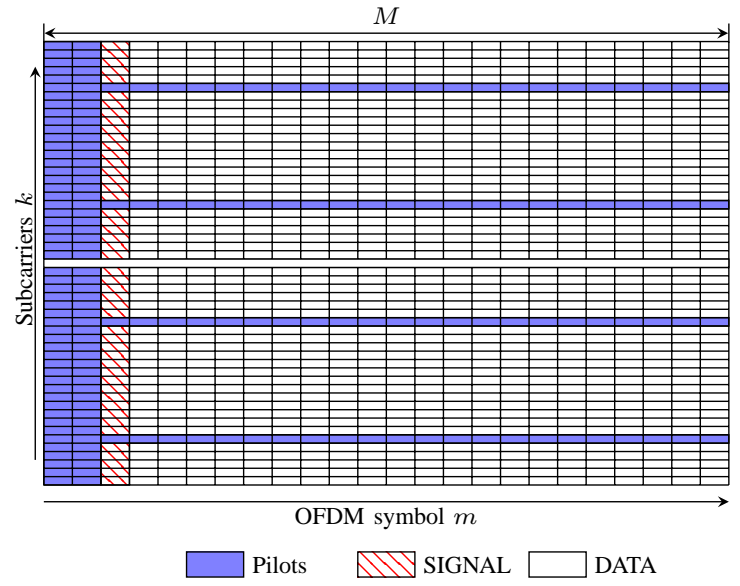

Fig. 2. A standard $802.11 \mathrm{p}$ frame in subcarrier-time grid showing the position of the pilots and the data symbols.

details of the various blocks in the transmitter refer $[6, \mathrm{Ch}$. 18].

A standard $802.11 \mathrm{p}$ frame (SF) in subcarrier-time grid is shown in Fig. 2. The frame begins with a sequence of ten identical waveforms spanning a duration of $2 T_{\mathrm{SYM}}$, called short training (not shown in the figure). The short training (ST) is used by the receiver for frame detection, synchronization, and automatic gain control. Two identical OFDM symbols referred to as long training (LT) symbols follow the short training and are mainly used for channel estimation and timing synchronization. The SIGNAL symbol carries the length of the packet and the MCS used for encoding the data bits. The SIGNAL symbol is encoded using the rate $1 / 2,(171,133)_{8}$ convolutional code without puncturing together with BPSK modulation. The SIGNAL field is followed by the OFDM symbols carrying the data. Every data OFDM symbol includes four pilot symbols referred to as comb pilots. The number of OFDM symbols in the SF beginning with the two LT symbols is denoted by $M$.

Assigning the data symbol positions to the set $\mathcal{D}$ and the pilot positions to the set $\mathcal{P}$, the frequency domain symbol mapped to the $k$ th subcarrier in the $m$ th OFDM symbol is given by

$$
x[m, k]=d[m, k]+p[m, k],
$$

where $d[m, k]$ and $p[m, k]$ are the data and the pilot symbols respectively, such that $p[m, k]=0, \forall[m, k] \notin \mathcal{P}$ and $d[m, k]=$ $0, \forall[m, k] \notin \mathcal{D}$. The first two identical OFDM symbols in LT have indices $m=0$ and $m=1$.

At the receiver, the CP of the received time domain OFDM symbol is discarded and an $N$-point DFT is performed to obtain the frequency domain symbols. Assuming perfect frequency and time synchronization, and that the length of the $\mathrm{CP}$ is longer than the channel impulse response, the received frequency domain symbols after the DFT operation are given by [19, Sec. 8.3]

$$
y[m, k]=h[m, k] x[m, k]+w[m, k],
$$

where $h[m, k]$ is the channel frequency response at the $k$ th subcarrier of the $m$ th OFDM symbol and $w[m, k]$ are inde-

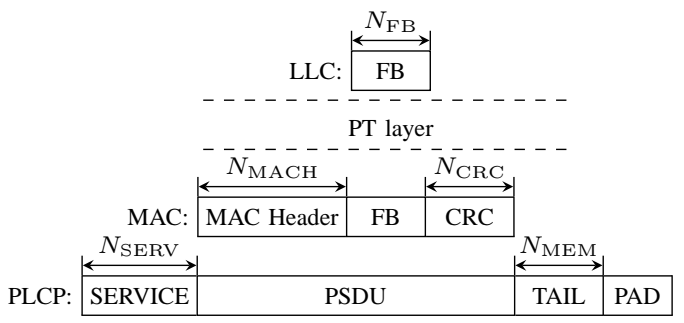

PHY: \begin{tabular}{l|l|l|l|}
\hline LT1 & LT2 & SIGNAL \\
\hline
\end{tabular}

DATA

Fig. 3. Diagram showing the flow of data bits from LLC layer to the PHY layer and the position of the proposed PT layer.

pendent and identically distributed complex Gaussian random variables with zero mean and variance $N_{0}$. The effect of intercarrier interference is neglected since the channel can be considered to be approximately time-invariant over the duration of one OFDM symbol.

\section{MODIFIED FRAME}

In this section, the proposed modified frame (MF) and its encoding process is described. The flow of the data from the logical link control (LLC) layer to the PHY layer is shown in Fig. 3. The LLC layer outputs a data unit referred to as the frame body (FB) of length $N_{\mathrm{FB}}$ bit. The FB is passed to the MAC layer, which adds a MAC header of length $N_{\mathrm{MACH}}=288 \mathrm{bit}^{1}$ and a cyclic redundancy check (CRC) checksum of length $N_{\mathrm{CRC}}=32$ bit (computed over the MAC header and the FB) to form the PHY layer convergence protocol (PLCP) service data unit (PSDU). In the PLCP sublayer, a SERVICE field of $N_{\mathrm{SERV}}=16 \mathrm{bit}$, a TAIL of $N_{\text {MEM }}$ zero bits to terminate the convolutional encoder in a known state, and padded zero bits are added to form the DATA unit of the OFDM frame. Since the MAC layer in 802.11p does not perform any encryption [7, Sec. 5.2.10] and hence no reordering of the input FB bits, the location of the FB bits in the DATA unit can be determined. The insertion of the additional training bits in the $F B$ to form the $M F$ is performed immediately after the LLC layer and before the MAC layer. We propose to introduce a new layer immediately after the LLC layer referred to as pseudo training (PT) layer to insert the training bits. This is easily done with a software/firmware update of the protocol stack residing above the MAC layer.

The training bits inserted in the PT layer are scrambled in the PHY layer. Since the scrambler is initialized with a pseudorandom seed, the training bits inserted in the PT layer are modified in a manner indeterminable at the PT layer. However, the pseudo-random sequence used to initialize the scrambler is estimated in the receiver. The estimated scrambler seed in combination with the inserted training sequence can be used by a modified receiver to recreate the output of the scrambler.

The rate $1 / 2,(171,133)_{8}$ convolutional encoder has a memory of $N_{\mathrm{MEM}}=6 \mathrm{bit}$. As a consequence, the two output bits of the convolutional encoder depend on the input bit and $N_{\mathrm{MEM}}$

\footnotetext{
${ }^{1}$ This is the length of the largest possible MAC header and is used in our results. The actual MAC header used can be smaller [6, Sec. 8.2].
} 


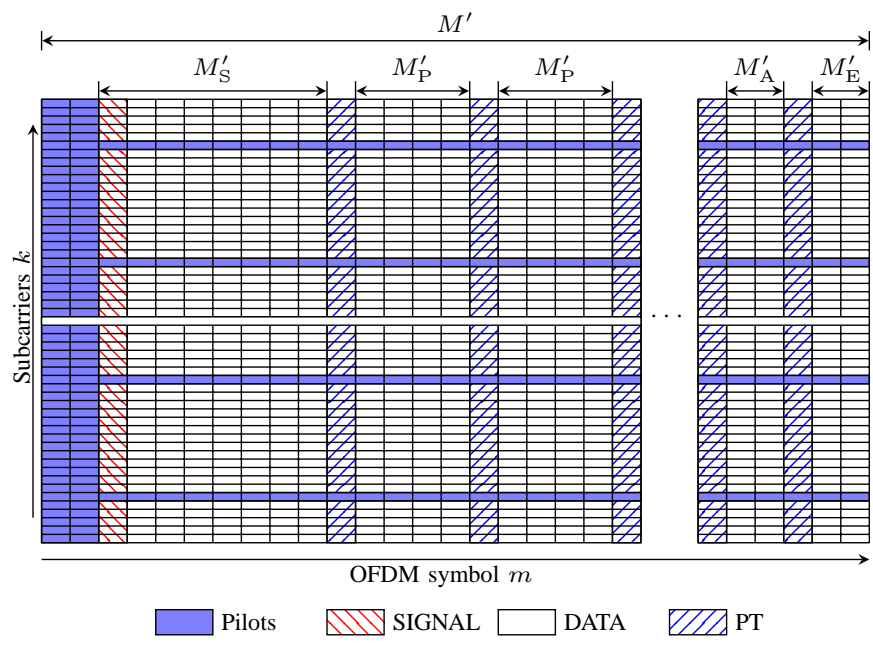

Fig. 4. A modified $802.11 \mathrm{p}$ frame in subcarrier-time grid showing the position of the pilots, the data symbols, and the inserted PT symbols.

previous input bits. To insert known training bits into the frame, the convolutional encoder is terminated in a known state before the training bits are fed to the convolutional encoder. The resulting sequence of output bits is therefore determined by the termination state and the input bits. Hence, inserting a known training sequence of any length into the frame requires $N_{\mathrm{MEM}}$ additional bits. Also, the encoded bits are interleaved over one OFDM symbol modifying the positions of the inserted bits. To minimize the overhead of termination and to insert training data which facilitates robust channel estimation for the frame, we insert training bits of length $N_{\text {DBPS }}$ (number of data bits per OFDM symbol) periodically in the frame. Inserting a complete OFDM training symbol requires the convolutional encoder to be terminated in a known state only once per OFDM training symbol resulting in smaller overhead. In addition, a complete OFDM symbol as training, similar to an LT symbol, has the advantage of measuring the frequency response across all the subcarriers.

Fig. 4 shows an example of the MF with periodically inserted OFDM training symbols. The inserted OFDM symbols will henceforth be referred to as PT symbols and the number of OFDM symbols between two periodically inserted PT symbols is denoted by $M_{\mathrm{P}}^{\prime}$, which is the design parameter of the MF. As shown in Fig. 4, the number of OFDM symbols between the LT symbols and the first PT is denoted by $M_{\mathrm{S}}^{\prime}$ which in some cases can be larger than $M_{\mathrm{P}}^{\prime}$ due to the insertion of the SIGNAL symbol, SERVICE field, and the MAC header by the layers below the PT layer. In fact, $M_{\mathrm{S}}^{\prime}$ is lower bounded by $\left\lceil\left(N_{\mathrm{SERV}}+N_{\mathrm{MACH}}+N_{\mathrm{MEM}}\right) / N_{\mathrm{DBPS}}\right\rceil+1$, where $\lceil x\rceil$ denotes the smallest integer larger or equal to $x$. When the packet is not long enough to enable the insertion of a PT with the specified $M_{\mathrm{P}}^{\prime}$, a PT is inserted at the end of the frame. Also, depending on the length $N_{\mathrm{FB}}$, the frame in the end may consist of less than $M_{\mathrm{P}}^{\prime}$ OFDM data symbols after the periodically inserted PT symbols. In that case, one additional PT is inserted after the periodically inserted PT at the end of the frame. The number of OFDM symbols between the final periodically inserted PT and the additional PT is denoted by

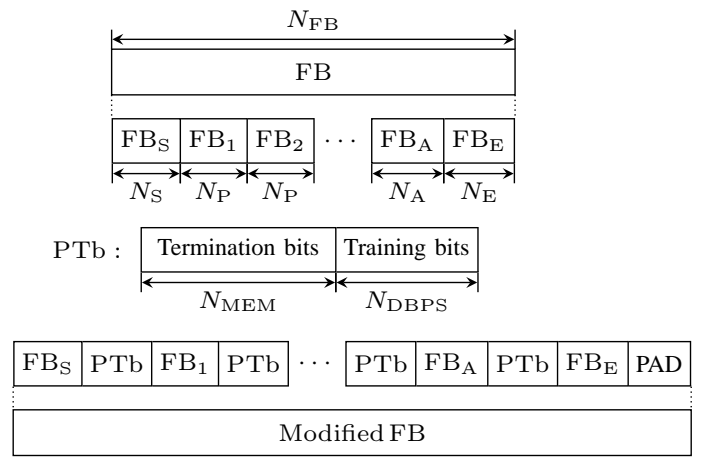

Fig. 5. Diagram showing the insertion of the training bits in the PT layer. The modified FB is made up of the original FB and the inserted PTb sequences.

$M_{\mathrm{A}}^{\prime}$. Since the CRC and the TAIL bits are appended to the end of the frame by the layers below the PT layer, the frame consists of $M_{\mathrm{E}}^{\prime}$ OFDM symbols after the final PT as shown in the figure. $M_{\mathrm{E}}^{\prime}$ is determined by $N_{\mathrm{FB}}, M_{\mathrm{P}}^{\prime}$, and the MCS used and is upper bounded by $\left\lceil\left(N_{\mathrm{CRC}}+N_{\mathrm{MEM}}\right) / N_{\mathrm{DBPS}}\right\rceil+1$. The number of OFDM symbols in the MF, beginning with the two LT symbols, is denoted by $M^{\prime}$.

To insert PT symbols as shown in Fig. 4, a transmitter uses a binary sequence of length $N_{\mathrm{MEM}}+N_{\mathrm{DBPS}}$ denoted by PTb which is known to a modified receiver. The first $N_{\text {MEM }}$ bit of the sequence terminate the encoder in a known state and the remaining $N_{\text {DBPS }}$ bit correspond to one OFDM training symbol. To ensure that the inserted bits result in a complete OFDM symbol as shown in the figure, the insertion has to be performed at specific positions, considering the interleaver and the other fields added. Since the scrambling is done in a random fashion unknown to the PT layer, the choice of bits in PTb cannot easily be optimized. For BPSK and QPSK modulations all choices give the same performance. In the numerical section, PTb was set to the all-zero sequence of length $N_{\mathrm{MEM}}+N_{\mathrm{DBPS}}$ bit. The procedure to insert training bits is described in the procedure InsertPT. The output of the procedure is a modified $F B$ which consists of the original FB and the inserted PTb sequences as illustrated in Fig. 5. The procedure is a modified version of the one described in our previous work [17]. The modification is related to the ability to insert a PT symbol at the end of short frames.

$M_{\mathrm{P}}^{\prime}$ can be fixed or made adaptive, in which case the transmitter chooses an $M_{\mathrm{P}}^{\prime}$ and includes its value in the transmitted frame. Currently the unused last nine bits in the SERVICE field can be used to convey $M_{\mathrm{P}}^{\prime}$ to the modified receiver in case $M_{\mathrm{P}}^{\prime}$ is adaptive (i.e., not known by the receiver). The reserved bit in the SIGNAL symbol and/or the presence of $M_{\mathrm{P}}^{\prime}$ in the last nine bits in the SERVICE field can be used to indicate a MF. If the SERVICE field and the reserved bit are not accessible to the PT layer during transmission, we instead propose to use a header in the PT layer to indicate the presence of a $\mathrm{MF}$ and the $M_{\mathrm{P}}^{\prime}$ used to the receiver. The header is added to the FB received from the LLC layer before it is fed to the procedure InsertPT.

We note that the modified FB has a size larger than the original FB due to the insertion of PTb sequences (and PT 

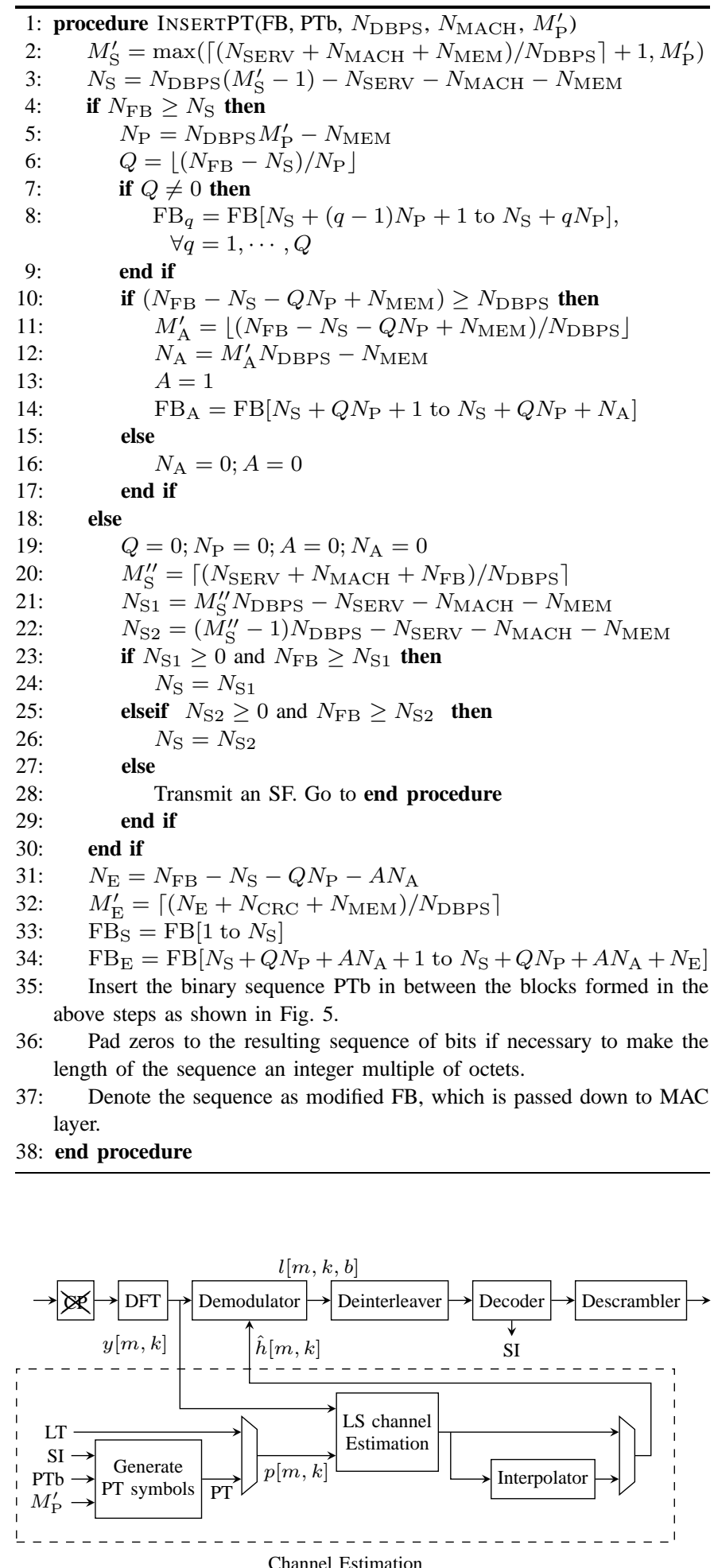

Fig. 6. Block diagram of the modified receiver.

layer header if present). As a consequence, the size of the FB fed to the procedure InsertPT has to be such that the size of the modified FB does not exceed the maximum allowed FB size (which is specified in [6]). However, this is not an issue for today's traffic safety applications, as proposed payloads are much smaller than the maximum allowed FB size.

\section{RECEIVER FOR MODIFIED 802.11P FRAME}

A receiver that utilizes the PT symbols for channel estimation is discussed in this section. There are many possible receiver structures that can utilize the PT symbols in different ways. The main contribution of the work is to provide more support for channel estimation by means of complementary training symbols introduced using a cross-layered approach and the focus is not on a specific channel estimation scheme.

Block diagram of an $802.11 \mathrm{p}$ receiver that can utilize the complementary training symbols is shown in Fig. 6. The frequency domain symbols $y[m, k]$ obtained after discarding the $\mathrm{CP}$ and performing the DFT operation are fed to the channel estimation block. The channel estimation block uses the received symbols and the pilots to obtain channel estimates $\hat{h}[m, k]$ for the entire frame. The received frequency domain symbols $y[m, k]$ and the channel estimates $\hat{h}[m, k]$ are then fed to a soft-demodulator. The soft-demodulator computes loglikelihood ratios (LLRs) of the encoded bits using max-log approximation. The max-log approximation LLR values are given by

$$
l[m, k, b]=\frac{1}{N_{0}} \sum_{u \in\{0,1\}}(-1)^{u} \min _{x_{i} \in \mathcal{X}_{b, u}}\left|y[m, k]-\hat{h}[m, k] x_{i}\right|^{2},
$$

where $\mathcal{X}_{b, u}$ is the set of constellation points whose $b^{\text {th }}$ bit is $u$. The scaling factor $1 / N_{0}$ can be ignored since it is constant for all the symbols. The LLRs are then deinterleaved and fed to the soft-input Viterbi decoder.

The pseudo-random scrambler initialization seed (SI) used to initialize the scrambler in the transmitter is necessary for reproducing the inserted PT symbols at the receiver side. The first 7 bit in the SERVICE field correspond to the SI and they are estimated with the assistance of channel estimates obtained using the LT symbols. Least-squares (LS) channel estimates are calculated using the two identical LT symbols and are averaged to obtain less noisy channel estimates given by

$$
\begin{aligned}
\hat{h}_{\mathrm{LT}}[k] & =\frac{1}{2}\left(\hat{h}_{\mathrm{LS}}[0, k]+\hat{h}_{\mathrm{LS}}[1, k]\right) \\
& =\frac{1}{2}\left(\frac{y[0, k]}{x[0, k]}+\frac{y[1, k]}{x[1, k]}\right) .
\end{aligned}
$$

The LS estimates can further be smoothed to lower the meansquared estimation error [20]. LS estimation is used in our work since it is readily implemented in hardware and does not require the knowledge of channel statistics.

The LLRs corresponding to the first several DATA OFDM symbols are computed using the LS channel estimates $\hat{h}_{\mathrm{LT}}[k]$ in (4) and the SERVICE field is decoded using Viterbi decoding with traceback. Traceback length of 120 trellis stages is used in the numerical results presented in Section VI. Using the estimated SI, PTb binary sequence, and $M_{\mathrm{P}}^{\prime}$, the inserted frequency domain training symbols are generated using the same procedure as in the transmitter. Subsequently, the LS estimates at the LT, comb pilots, and PT positions are computed and fed to the interpolator.

A blockwise linear minimum mean squared error (LMMSE) interpolator that utilizes PT symbols is described in our previous work [17]. The blockwise LMMSE interpolator achieves 
FER performance close to the case with perfect CSI for suitably chosen $M_{\mathrm{P}}^{\prime}$. An LMMSE interpolator has two main drawbacks, i) it relies on autocorrelation functions of the underlying channels and $N_{0}$, and ii) it involves operations on large matrices and is not suitable for hardware implementation.

To demonstrate the hardware implementation feasibility of the proposed pilot insertion scheme, we have chosen to perform channel estimation using estimate and hold, and linear interpolation (described in the following subsections), which are easily implemented in hardware.

\section{A. Blockwise Estimate and Hold}

In the blockwise estimate and hold (PT Hold) method, LS channel estimates are computed at the PT symbol positions and are used as the channel estimates for the data symbols until the next PT is received. This is the simplest method for utilizing the PT symbols for improved channel estimation and it does not require additional buffering of received data OFDM symbols. The estimate and hold channel estimates for the $m^{\text {th }}$ OFDM symbol sandwiched between two consecutive PT symbols with indices $m=I$ and $m=J$, respectively, are given by

$$
\hat{h}[m, k]=\hat{h}_{\mathrm{LS}}[I, k], \forall m=I, I+1, \cdots, J-1 .
$$

\section{B. Blockwise Linear Interpolation}

In the blockwise linear interpolation (PT Linear) method, linear interpolation is performed between the two consecutive PT symbols to obtain the channel estimates for the data symbols sandwiched between two consecutive PT symbols. In contrast to LMMSE, linear interpolation has the advantage of not requiring the channel autocorrelation functions or $N_{0}$. Linearly interpolated channel estimates for the $m^{\text {th }}$ OFDM symbol sandwiched between two consecutive PT OFDM symbols with indices $m=I$ and $m=J$, respectively, is given by

$$
\begin{aligned}
& \hat{h}[m, k]=(1-u) \hat{h}_{\mathrm{LS}}[I, k]+u \hat{h}_{\mathrm{LS}}[J, k], \\
& \text { where } u=\frac{m-I}{J-I} \text { and } \forall m=I, I+1, \cdots, J-1 .
\end{aligned}
$$

For the final block of $M_{\mathrm{E}}^{\prime}$ OFDM symbols, the channel estimates corresponding to the last PT are used.

Simulation results show that robust channel estimates can be obtained using linear interpolation for carefully chosen $M_{\mathrm{P}}^{\prime}$. The PT Linear scheme requires the buffering of the received data OFDM symbols sandwiched between two consecutive PT symbols.

\section{Overhead}

The PT symbols inserted in the MFs introduce additional overhead and hence result in loss of spectral efficiency. Overhead due to PT insertion is a function of $N_{\mathrm{FB}}, M_{\mathrm{P}}^{\prime}$ and the MCS used. An MF consists of $(1+Q+A)$ extra OFDM symbols compared to the SF carrying same information payload. Hence, the total number of bits before the PHY layer

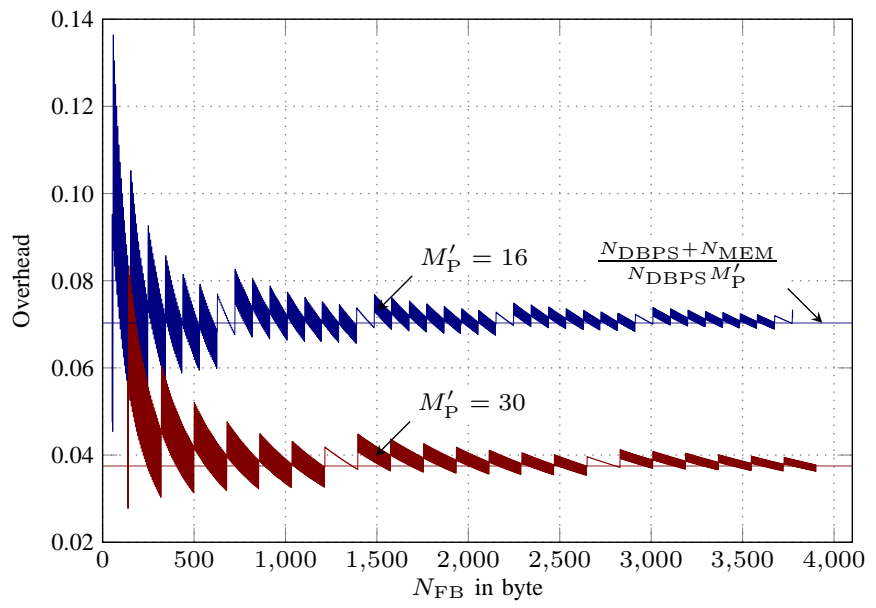

Fig. 7. Overhead due to PT insertion

zero padding in the $\mathrm{SF}$ and $\mathrm{MF}$, denoted by $N_{\mathrm{SF}}$ and $N_{\mathrm{MF}}$, respectively, are

$$
\begin{aligned}
& N_{\mathrm{SF}}=N_{\mathrm{SERV}}+N_{\mathrm{MACH}}+N_{\mathrm{FB}}+N_{\mathrm{CRC}}+N_{\mathrm{MEM}}, \\
& N_{\mathrm{MF}}=N_{\mathrm{SF}}+(1+Q+A) \cdot\left(N_{\mathrm{DBPS}}+N_{\mathrm{MEM}}\right) .
\end{aligned}
$$

The additional overhead due to inserted PT symbols in an MF is given by

$$
O=\frac{\left\lceil N_{\mathrm{MF}} / N_{\mathrm{DBPS}}\right\rceil-\left\lceil N_{\mathrm{SF}} / N_{\mathrm{DBPS}}\right\rceil}{5+\left\lceil N_{\mathrm{SF}} / N_{\mathrm{DBPS}}\right\rceil},
$$

where the constant 5 represents the number of OFDM symbols equivalent to the short training, LT, and SIGNAL symbols. The effective bit rates of the SFs and the MFs denoted by $R_{\mathrm{SF}}$ and $R_{\mathrm{MF}}$, respectively, are related as $R_{\mathrm{SF}}=(1+O) R_{\mathrm{MF}}$.

Fig. 7 shows the overhead due to PT symbols in the MFs for the MCS with QPSK and code rate $1 / 2$. The curves have been terminated when the length of the modified FB exceeds the maximum allowed length of 4096 byte. The additional overhead due to inserted PTs is approximately equal to $\left(N_{\mathrm{DBPS}}+N_{\mathrm{MEM}}\right) /\left(N_{\mathrm{DBPS}} M_{\mathrm{P}}^{\prime}\right)$ for long frames for which the overhead due to the ST, LT, and SIGNAL symbols is negligible. The approximation is also shown in the figure. As seen from the figure, the overhead for $M_{\mathrm{P}}^{\prime}=16$ and an FB of approximately 400 byte varies between 6 and $8 \%$. In other words, the MF duration is 6 to $8 \%$ longer than the SF duration, resulting in 6 to $8 \%$ lower spectral efficiency.

\section{HARDWARE IMPLEMENTATION}

The receiver described in Section IV for utilizing the complementary training symbols has been implemented on the hardware platform HIRATE. The architecture of the HIRATE platform is shown in Fig. 8. HIRATE is based on a Gidel Procstar III evaluation board with four Altera Stratix FPGAs (260E), which is extended by a custom made daughter board. Reference clock (Ref. CLK), sample clock (Samp. CLK), local oscillators (LOs), low noise amplifier (LNA) with down mixers, power amplifier (PA) with modulators, and low pass filters (LPFs) are designed as plug-on modules for placement on the daughter board to allow flexibility in the system 


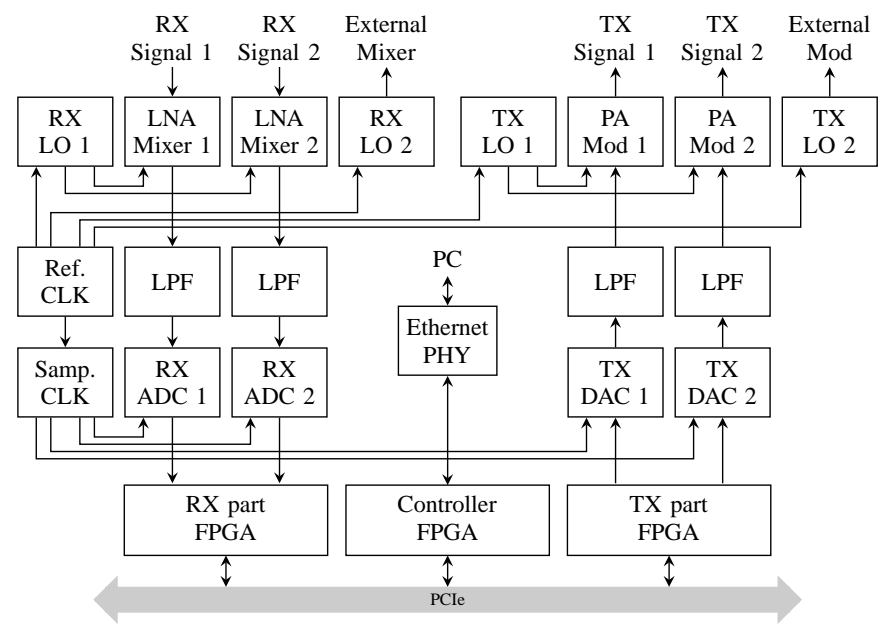

Fig. 8. HIRATE architecture.

configuration. The output from local oscillators can be fed to external down mixers and modulators. The daughter board includes two 16 bit digital to analog converters (DACs) with a maximum sampling rate of $250 \mathrm{MS} / \mathrm{s}$ and two 8 bit analog to digital converters (ADCs) with a maximum sampling rate of $500 \mathrm{MS} / \mathrm{s}$. The transceiver analog chain is configured using the DACs, ADCs, and the plug-on modules. The DACs and ADCs are interfaced with the TX and RX FPGAs, respectively, where the baseband digital processing is performed. The platform can be configured to operate in single input single output (SISO) or $2 \times 2$ multiple input multiple output (MIMO) mode using the plug-on modules. The platform is configured in SISO mode in our implementation. Ethernet and PCIe interfaces enable communication between FPGAs and a PC.

The design flow for implementing baseband processing is based on Altera DSP builder which allows graphical development and implementation of the baseband transceiver using Simulink and MATLAB. Altera DSP builder provides a library of design elements in Simulink that can be used to build the necessary signal processing algorithms.

Frame detection, frequency offset computation, and timing detection are performed using the short training sequence based on the algorithms proposed in [21].

FER tests have been performed with the hardware implementation and compared with computer simulations. Although the hardware implementation uses the same algorithms as in the computer simulations, differences exist that create a mismatch in the results. Some of the major differences between the computer simulation and the HIRATE implementation are:

- Signals in the computer simulations have a 64 bit wide floating point representation, whereas the signals in the hardware implementation use fixed point representation with varying bit widths. DAC and ADCs are not present in the computer simulations, whereas HIRATE uses DAC and ADCs of 16 and 8 bit, respectively.

- The computer simulations are performed in baseband at a sampling rate of $10 \mathrm{MS} / \mathrm{s}$. In the hardware implementation, baseband samples at $10 \mathrm{MS} / \mathrm{s}$ are upsampled to 160 MS/s and filtered using an FIR filter at the transmitter

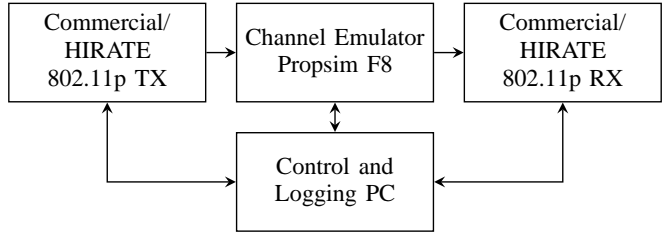

Fig. 9. Measurement setup used for performing FER tests.

before they are fed to the DAC. At the receiver, the output of the ADC sampled at $160 \mathrm{MS} / \mathrm{s}$ is filtered using an FIR filter and then downsampled to $10 \mathrm{MS} / \mathrm{s}$.

- LLRs are not quantized in the computer simulations, whereas LLRs are quantized to 16 bit before they are fed to the Viterbi decoder in the hardware implementation.

- Carrier frequency offsets are absent in the computer simulations since all the processing is done in baseband. The same reference clock is used for the HIRATE transmitter and receiver to reduce the carrier frequency mismatch.

\section{NumericAl Results AND Discussion}

Real time FER measurements have been performed by interfacing HIRATE and commercially available $802.11 \mathrm{p}$ transceivers with a channel emulator. The measurement setup is shown in Fig. 9. The RF output and inputs of the transceiver are connected to an Anite Propsim F8 channel emulator that allows the emulation of multipath wireless channels with user defined power delay profiles (PDPs) and Doppler spectrums. A computer interfaced with the emulator and the transceivers is used to configure the PHY layer parameters of the transceivers, control the channel emulator, and log the measurement data. The exact channel models used in the computer simulations were used to emulate the channels for hardware measurements. There might be some differences in the method and the precision used by the channel emulator to emulate the channels.

\section{A. Channel Models}

Tapped-delay line channel models have been used to perform FER measurements. In the first two channel models described below, the tap gains are independent zero mean complex Gaussian (Rayleigh distributed) with autocorrelation function $\alpha_{l} \mathrm{~J}_{0}(2 \pi(v / \lambda) t)$, where $v$ is the relative speed between the transmitter and the receiver, $\lambda$ is the wavelength of the electromagnetic carrier wave of frequency $f_{c}=5.9 \mathrm{GHz}$, $\alpha_{l}$ is the power in the lth tap, and delay of the lth tap is represented by $\tau_{l}$.

1) Exponentially decaying PDP (EXP): The power in the $l$ th tap for this model is given by $\alpha_{l}=K \mathrm{e}^{-\tau_{l} / \tau_{\mathrm{RMS}}}$, where $\tau_{\mathrm{RMS}}$ is the root-mean squared (RMS) delay spread of the continuous and infinitely long exponentially decaying PDP, and $K$ is the normalization factor such that $\sum_{l=0}^{L-1} \alpha_{l}=1$. The model consists of $L=15$ taps spanning the delay from 0 to $1.4 \mu \mathrm{s}$ with a tap spacing of $0.1 \mu \mathrm{s}$. The effective RMS delay spread denoted by $\tau_{\text {ERMS }}$ is different from $\tau_{\text {RMS }}$ due to truncation and sampling. An RMS delay spread of $\tau_{\mathrm{RMS}}=0.4 \mu \mathrm{s}$ is used in the channel model, which results in an effective RMS delay spread of $\tau_{\text {ERMS }}=0.322 \mu \mathrm{s}$. The 
TABLE I

PDP OF THE UMI CHANNEL.

\begin{tabular}{|c|c|c|c|c|c|c|}
\hline$\tau_{l}$ in $\mathrm{ns}$ & 0 & 200 & 300 & 700 & 800 & 900 \\
\hline$\alpha_{l}$ in $\mathrm{dB}$ & 0 & -2.7 & -1.3 & -4.3 & -6.0 & -8.0 \\
\hline
\end{tabular}

TABLE II

PDPS AND DOPPLER PROFILES OF C2C CHANNELS.

\begin{tabular}{|c|c|c|c|c|||c|c|c|c|}
\hline Channel & \multicolumn{4}{|c||}{ Highway LoS } & \multicolumn{3}{c|}{ Urban Approaching LoS } \\
\hline$\tau_{l}$ in $\mathrm{ns}$ & 0 & 100 & 167 & 500 & 0 & 117 & 183 & 333 \\
\hline$\alpha_{l}$ in $\mathrm{dB}$ & 0 & -10 & -15 & -20 & 0 & -8 & -10 & -15 \\
\hline$f_{\mathrm{D}}$ in $\mathrm{Hz}$ & 0 & 689 & -492 & 886 & 0 & 236 & -157 & 492 \\
\hline \hline Channel & \multicolumn{5}{|c||}{ Crossing NLoS } & \multicolumn{5}{c|}{ Highway NLoS } \\
\hline$\tau_{l}$ in ns & 0 & 267 & 400 & 533 & 0 & 200 & 433 & 700 \\
\hline$\alpha_{l}$ in $\mathrm{dB}$ & 0 & -3 & -5 & -10 & 0 & -2 & -5 & -7 \\
\hline$f_{\mathrm{D}}$ in $\mathrm{Hz}$ & 0 & 295 & -98 & 591 & 0 & 689 & -492 & 886 \\
\hline
\end{tabular}

EXP channel exhibits high frequency selectivity due to the large delay spread.

2) Urban Micro (UMi): In this channel model we use a simplified version of the urban micro (UMi) channel model proposed by 3GPP [22, Table 8.2-1], where the taps in each cluster have been combined into a single tap with the aggregate power and the delays in a cluster are averaged to obtain the tap delay. The PDP of the simplified UMi channel model used for the FER measurements is shown in Table I.

3) Channels from Car-2-Car Communication Consortium $(C 2 C)$ : Traffic scenario based V2V channel models based on measurements have been developed in the Car-2-Car Communication Consortium [23]. The PDPs of the channels and the Doppler frequencies of the taps are given in Table II. Line-ofsight and non line-of-sight are denoted by LoS and NLoS, respectively. The paths at delay $\tau_{l}=0$ are static and the following paths have a Doppler profile with a single Doppler frequency at specified frequency $f_{\mathrm{D}}$ in contrast to the EXP and UMi channel models where all the taps are time-varying.

\section{B. Compatibility Test}

Compatibility of the MF with a standard receiver is verified using an off the shelf $802.11 \mathrm{p}$ transceiver whose higher layers are updated to allow insertion and removal of PTb sequences. The off the shelf transceiver was able to decode MFs and pass the data to the higher layers. The bits corresponding to the complementary training bits were removed from the packet to retrieve the data. Also, MFs generated using the proposed algorithm were transmitted with the off the shelf $802.11 \mathrm{p}$ transceiver and the receiver implemented in HIRATE utilized the complementary training bits to perform robust channel estimation. The result of these tests verifies that the proposed scheme is compatible with the standard 802.11p transceivers with the additional requirement that a software/firmware update in the higher layers is able to remove/insert training bits.

\section{Frame Error Rate Measurements}

FER performance obtained with real time measurements and computer simulations are discussed in this section. The MCS with QPSK mapping and code rate 1/2 [6, Table 18.4] is adopted for the safety applications [24, Sec. 5.3] and hence all the FER results in this section correspond to this MCS. A frame is considered to be in error if the appended CRC checksum does not agree with the checksum computed in the receiver.

The signal-to-noise ratio (SNR) used in the FER performance plots is the average subcarrier SNR. The SNR for the measurements is estimated using the two received LT OFDM symbols, which contain 52 subcarriers with data or pilot symbols of equal energy. The average subcarrier SNR for these cases is estimated using

$$
\left(\frac{E_{\mathrm{s}}}{N_{0}}\right)_{\text {frame }}=\frac{64}{52}\left(\frac{\sum_{k=0}^{63}|y[0, k]|^{2}}{\frac{1}{2} \sum_{k=0}^{63}|y[1, k]-y[0, k]|^{2}}-1\right),
$$

for every detected frame and then averaged over all frames to obtain $E_{\mathrm{s}} / N_{0}$. For fairness, in the simulations used for comparison with the hardware measurements, the SNR has also been estimated using the same procedure. Note that (7) is an approximation under the assumption that the channel is constant between the two LT OFDM symbols.

FER performance tests have been performed for varying frame sizes since frames of length 200 to 800 byte have been mentioned in the vehicular communications literature. Frame body sizes approximately equal to $N_{\mathrm{FB}}=150, N_{\mathrm{FB}}=500$, and $N_{\mathrm{FB}}=1000$ byte have been chosen for our tests. Slightly different frame sizes have been chosen for different $M_{\mathrm{P}}^{\prime}$ to avoid insertion of a nonperiodic PT at the end of the frame such that $M_{\mathrm{S}}^{\prime}=M_{\mathrm{P}}^{\prime}, M_{\mathrm{E}}^{\prime}=1$, and an additional PT after periodic insertion is not required (see Fig. 4). Parameters that occur frequently in the discussion of the numerical results are presented in Table III.

TABLE III

FREQUENTLY OCCURRING PARAMETERS IN THE NUMERICAL RESULTS

\begin{tabular}{cc}
\hline Parameter & Symbol \\
\hline Length of the frame body & $N_{\mathrm{FB}}$ \\
No. of OFDM symbols in an SF & $M$ \\
No. of OFDM symbols in an MF & $M^{\prime}$ \\
No. of OFDM symbols between & $M_{\mathrm{P}}^{\prime}$ \\
periodically inserted PTs & $v$ \\
Relative vehicular velocity &
\end{tabular}

Fig. 10 shows the FER results obtained using computer simulations for an MF with $N_{\mathrm{FB}}=147$ byte using the EXP channel with $v=100 \mathrm{~km} / \mathrm{h}$ and $M_{\mathrm{P}}^{\prime}=16$. The block based LMMSE channel estimation method (BMMSE) described in our previous work [17] provides FER performance very close to the case with perfect CSI for an SF with same $N_{\mathrm{FB}}$. The LT Hold channel estimation scheme that estimates the channel at the beginning of the frame using the LT OFDM symbols and uses it for the rest of the frame performs poorly due to the highly time- and frequency-varying nature of the channel. The PT Hold channel estimation technique provides improved performance due to the periodic nature of the inserted PT symbols. The FER performance is further improved in the case of the PT Linear technique and the performance improves with increasing SNR. In contrast to the BMMSE method, the PT Linear technique involves simple linear interpolation as 


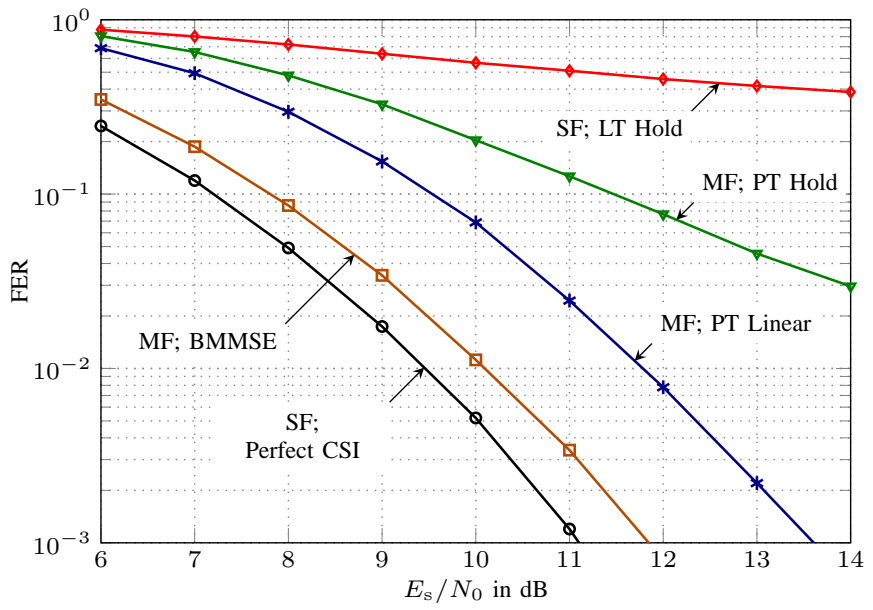

Fig. 10. Simulation FER results of SFs and MFs using different channel estimation techniques in the EXP channel with relative vehicular velocity $v=100 \mathrm{~km} / \mathrm{h}$. Frames carry an FB of length $N_{\mathrm{FB}}=147$ byte and $M_{\mathrm{P}}^{\prime}=16$ for the MFs.

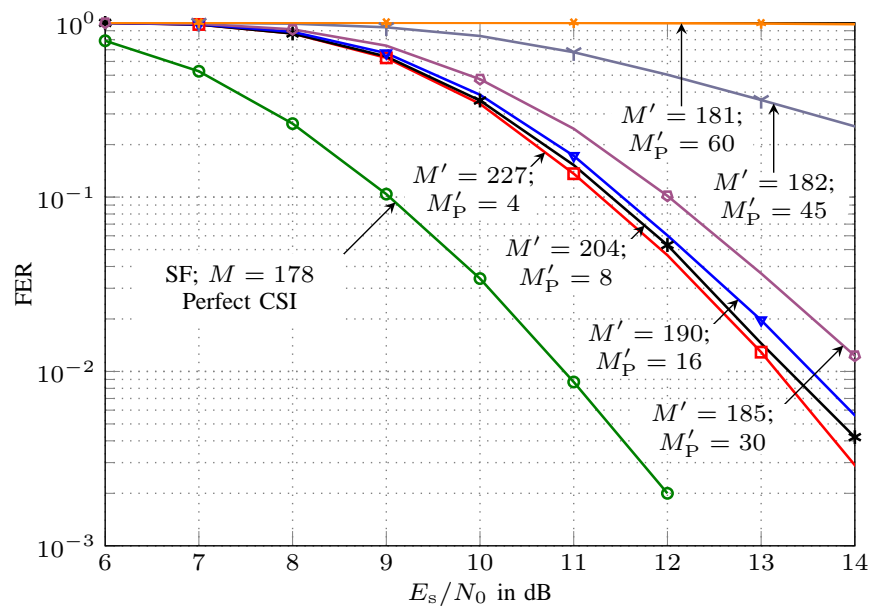

Fig. 11. Simulation FER results of MFs using PT Linear channel estimation scheme for different $M_{\mathrm{P}}^{\prime}$ in the EXP channel with $v=200 \mathrm{~km} / \mathrm{h}$.

described in Section IV-B. The PT Linear estimation method has been implemented on the HIRATE platform and will be the focus of the FER performance measured using the hardware implementation.

The number of OFDM symbols between two consecutive PT symbols, $M_{\mathrm{P}}^{\prime}$, is a design parameter of the MF. Fig. 11 shows the FER performance of the PT Linear channel estimation scheme for different values of $M_{\mathrm{P}}^{\prime}$ in EXP channel with $v=200 \mathrm{~km} / \mathrm{h}$. An SF with $N_{\mathrm{FB}}=1004$ byte corresponding to $M=178$ is transmitted, and the lengths of the corresponding MFs are $M^{\prime}=\{227,204,190,185,182,181\}$ for $M_{\mathrm{P}}^{\prime}=\{4,8,16,30,45,60\}$, respectively. The FER of the SF with LT Hold scheme is close to 1 for all SNRs and is therefore not shown in the figure. The FER performance of the PT Linear scheme starts to degrade with $M_{\mathrm{P}}^{\prime} \geq 30$ and the FER is close to 1.00 for $M_{\mathrm{P}}^{\prime}=60$. As seen in Fig. 11, the FER performance does not improve substantially when $M_{\mathrm{P}}^{\prime}$ is reduced from 16 to 4 . This is due to the fact that, when the consecutive PT symbols are placed such that the channel

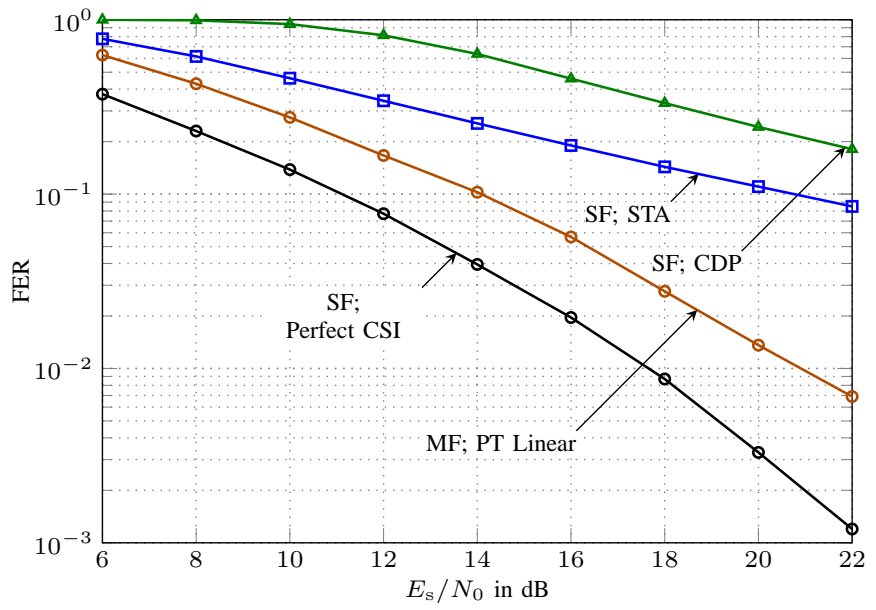

Fig. 12. Comparison of STA and CDP schemes with the proposed MFs using PT Linear scheme. Frames carry an FB of length $N_{\mathrm{FB}}=528$ byte and $M_{\mathrm{P}}^{\prime}=16$ for the MF.

variation between them is approximately linear, the mean squared channel estimation error in the PT Linear scheme is dominated by the estimation error of the LS estimates at the PT symbol positions and the error due to linear interpolation is negligible. As a consequence, the mean squared channel estimation error does not reduce significantly when $M_{\mathrm{P}}^{\prime}$ is reduced from 16 to 4 , and the FER performance does not improve substantially. Therefore, we have used $M_{\mathrm{P}}^{\prime}=16$ in the experiments performed using the hardware implementation.

In Fig. 12, the performance of the CDP and the STA schemes proposed in [9] and [8], respectively, have been compared to the PT Linear scheme with $M_{\mathrm{P}}^{\prime}=16$. For the STA scheme, $\alpha=2$ and $\beta=2$ is used [8]. The V2V urban canyon oncoming channel model in [25, Table 2] is used and the frames carry an FB of length $N_{\mathrm{FB}}=528$ byte. The FER of the SF with LT Hold scheme is close to 1.00 for all SNRs and is not shown in the figure. As seen in the figure, STA and CDP schemes show considerable performance improvement over the LT Hold scheme. However, the performance of the CDP and STA schemes is far from the case with perfect CSI. Moreover, both schemes are outperformed by PT linear. This is quite obvious, as the STA and CDP schemes use SFs and, in contrast to PT linear, cannot benefit from the extra pilots in MFs.

Fig. 13 shows the FER performance of MFs using PT Linear channel estimation in EXP channel. Frames carry an FB of length $N_{\mathrm{FB}}=147$ byte and $M_{\mathrm{P}}^{\prime}=16$. The results show that the FER performance of the HIRATE receiver follows the simulation results with an approximately constant SNR offset. The output power of the channel emulator was varied to obtain different SNRs and the SNRs used in the plot are the average measured SNRs according to (7). The frame detection algorithm, the sampling time algorithm, and the filters implemented in the HIRATE transceiver are included in the computer simulations. Only the detected frames are considered for measuring the FER to exclude the influence of the frame detection algorithms on the FER performance. The difference in FER performance between the computer simulations and the 


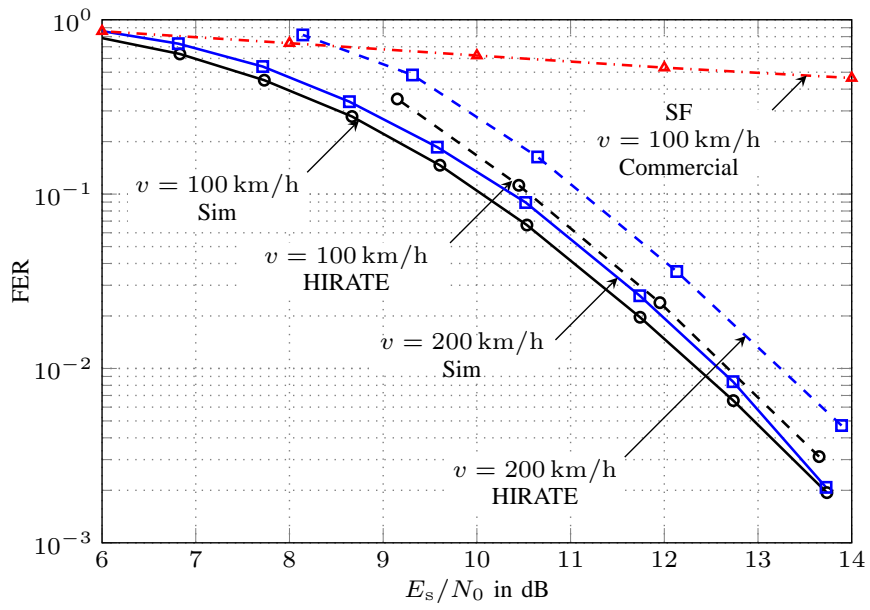

Fig. 13. FER results of MFs with $N_{\mathrm{FB}}=147$ byte and $M_{\mathrm{P}}^{\prime}=16$ using PT Linear channel estimation in EXP channel. The measured results follow the simulation results with an SNR offset. The FER performance of a commercial transceiver using SFs is also shown.

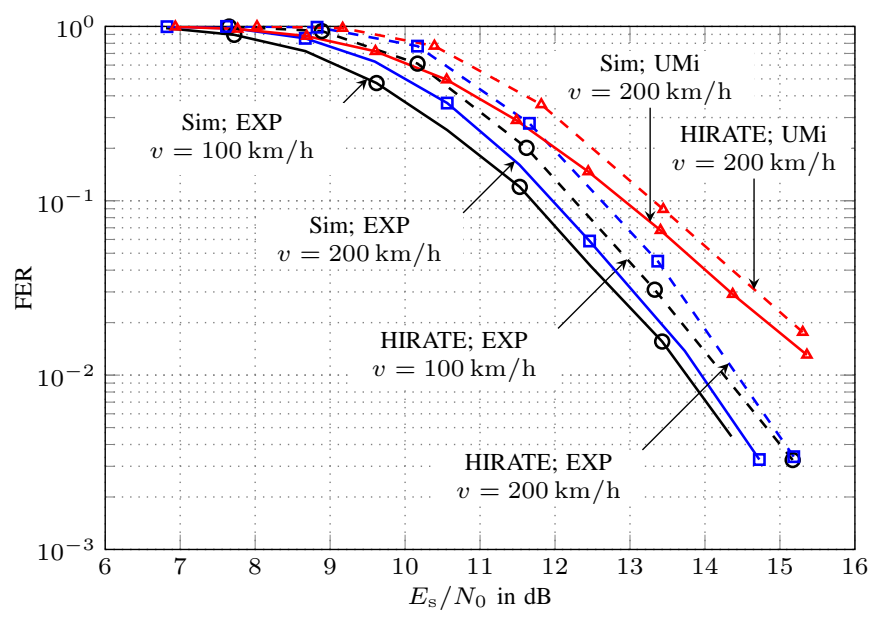

Fig. 14. FER results of MFs with $M_{\mathrm{P}}^{\prime}=16$ and $N_{\mathrm{FB}}=1004$ byte using PT Linear channel estimation in EXP and UMi channels. The measured results follow the simulation results with an SNR offset.

hardware measurements is due to the differences discussed in Section V. In the FPGA implementation, different signals at different blocks of the transceiver have varying precisions and hence the performance of the algorithms suffers from varying quantization effects. It is not straight forward to quantitatively compute the effective degradation in FER performance due to all the individual losses caused by quantization. All the algorithms implemented in the hardware have to be reproduced with the exact precisions in computer simulations and compared with the measurements for fairness. Also, all the effects of analog components have to be included in the computer simulations. This is not in the scope of our work. The measured FER performance of a commercial 802.11p transceiver in EXP channel is also shown in the figure as a reference. The FER decreases slowly with SNR and this is most likely due to the nonrobust channel estimation based on LT symbols alone.

Fig. 14 shows the FER performance of the MFs with

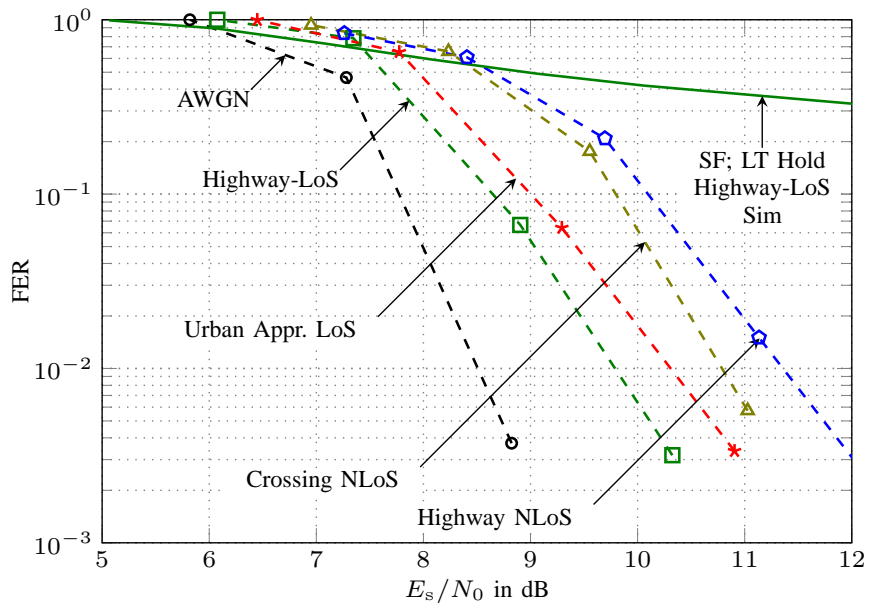

Fig. 15. FER results of HIRATE receiver for MFs with $M_{\mathrm{P}}^{\prime}=30$ and $N_{\mathrm{FB}}=1032$ byte using PT Linear channel estimation in AWGN and C2C channels (dashed curves). The simulation FER result of SFs in Highway-LoS channel using LT Hold scheme is also shown.

$M_{\mathrm{P}}^{\prime}=16$ and $N_{\mathrm{FB}}=1004$ byte in EXP and UMi channels using PT Linear channel estimation. Similar to the results in Fig. 13, FER performance of the HIRATE receiver follows the simulation results with an approximately constant SNR offset. Simulations and HIRATE measurements have also been performed for MFs with $M_{\mathrm{P}}^{\prime}=30$ and the HIRATE results follow the simulation results in a similar fashion (not included here). The commercial transceiver was not able to decode SFs with the same $N_{\mathrm{FB}}$ in EXP and UMi channels with $v=100 \mathrm{~km} / \mathrm{h}$ and $v=200 \mathrm{~km} / \mathrm{h}$.

In Fig. 15, the FER performance of the HIRATE receiver for the MFs with $M_{\mathrm{P}}^{\prime}=30$ and $N_{\mathrm{FB}}=1032$ byte in AWGN and C2C channels using PT Linear channel estimation is shown. The steeper slopes of the FER vs. SNR curves in case of the C2C channels is due to the static nature of the $\tau_{l}=0$ tap. The simulation result in highway LoS channel for SFs (with the same FB size) using LT Hold channel estimation is also shown. In this case, the FER decreases slowly with SNR in comparison to the HIRATE performance.

\section{CONCLUSION}

In this paper we have shown the hardware implementation feasibility of the proposed cross-layered pilot scheme and its compatibility with the standard 802.11 transceivers. Linear interpolation is chosen for channel estimation, since it is readily implemented in hardware. It is seen from the numerical results that a linear interpolation scheme in combination with suitably chosen separation between PT symbols delivers improved FER performance. More sophisticated channel estimation schemes with reasonable hardware implementation complexity that utilize the inserted PT symbols can be implemented to further improve the FER performance.

The proposed pilot insertion scheme is not specific to a channel estimation scheme and the complementary training symbols can be utilized by receivers using decision feedback or iterative decoding strategies to either improve the accuracy or reduce the complexity of these channel estimation schemes. 
Layers above the MAC layer can decide the period of the inserted PT symbols. As an example, safety critical messages or messages with higher priority could have higher pilot density and the density can be decided directly by the higher layer application in the transmitter and adaptively communicated to the receiver.

Compatibility of the proposed scheme allows the coexistence of standard transceivers that are already deployed and the modified receivers that can exploit the complementary training symbols to improve the performance.

\section{ACKNOWLEDGMENT}

The authors would like to thank Panagiotis Paschalidis for enabling the collaboration between Chalmers and HHI through the COST IC 1004 action. We would also like to thank Andreas Kortke, Thomas Kühne, and Leszek Koschel for the development of the HIRATE platform.

\section{REFERENCES}

[1] "Intelligent transport systems (ITS); vehicular communications; basic set of applications; definitions," ETSI TR 102638 (V1.1.1), 2009.

[2] C. Mecklenbräuker, A. F. Molisch, J. Karedal, F. Tufvesson, A. Paier, L. Bernadó, T. Zemen, O. Klemp, and N. Czink, "Vehicular channel characterization and its implications for wireless system design and performance," Proceedings of the IEEE, vol. 99, no. 7, pp. 1189-1212, July 2011.

[3] L. Bernadó, T. Zemen, F. Tufvesson, A. F. Molisch, and C. Mecklenbräuker, "Delay and Doppler spreads of nonstationary vehicular channels for safety-relevant scenarios," IEEE Transactions on Vehicular Technology, vol. 63, no. 1, pp. 82-93, Jan. 2014.

[4] E. G. Ström, "On medium access and physical layer standards for cooperative intelligent transport systems in Europe," Proceedings of the IEEE, vol. 99, no. 7, pp. 1183-1188, July 2011, Invited paper.

[5] J. B. Kenney, "Dedicated short-range communications (DSRC) standards in the United States," Proceedings of the IEEE, vol. 99, no. 7, pp. 11621182, July 2011, Invited paper.

[6] "Wireless LAN medium access control (MAC) and physical layer (PHY) specifications," IEEE Std 802.11-2012, pp. 1-2793, 2012.

[7] "Wireless LAN medium access control (MAC) and physical layer (PHY) specifications, Amendment 6: Wireless access in vehicular environments," IEEE Std 802.11p-2010, pp. 1-51, 2010.

[8] J. Fernandez, K. Borries, L. Cheng, B. Kumar, D. Stancil, and F. Bai, "Performance of the $802.11 \mathrm{p}$ physical layer in vehicle-to-vehicle environments," IEEE Transactions on Vehicular Technology, vol. 61, no. 1, pp. 3-14, Jan. 2012.

[9] Z. Zhao, X. Cheng, M. Wen, B. Jiao, and C.-X. Wang, "Channel estimation schemes for IEEE $802.11 \mathrm{p}$ standard," IEEE Intelligent Transportation Systems Magazine, vol. 5, no. 4, pp. 38-49, Oct. 2013.

[10] T. Kella, "Decision-directed channel estimation for supporting higher terminal velocities in OFDM based WLANs," in IEEE Global Telecommunications Conference, San Francisco, CA, USA, Dec. 2003, pp. 13061310.

[11] T. Zemen, L. Bernadó, N. Czink, and A. F. Molisch, "Iterative timevariant channel estimation for $802.11 \mathrm{p}$ using generalized discrete prolate spheroidal sequences," IEEE Transactions on Vehicular Technology, vol. 61, no. 3, pp. 1222-1233, Mar. 2012.

[12] O. Goubet, G. Baudic, F. Gabry, and T. J. Oechtering, "Low-complexity scalable iterative algorithms for IEEE $802.11 \mathrm{p}$ receivers," IEEE Transactions on Vehicular Technology, vol. 64, no. 9, pp. 3944-3956, Sept. 2015.

[13] Z. Li, F. Bai, J. A. Fernandez, and B. V. K. Vijaya Kumar, "Tentpoles scheme: A data-aided channel estimation mechanism for achieving reliable vehicle-to-vehicle communications," IEEE Transactions on Wireless Communications, vol. 14, no. 5, pp. 2487-2499, May 2015.

[14] R. Grünheid, H. Rohling, J. Ran, E. Bolinth, and R. Kern, "Robust channel estimation in wireless LANs for mobile environments," in IEEE Vehicular Technology Conference, Vancouver, BC, Canada, Sept. 2002, pp. 1545-1549.
[15] W. Cho, S. I. Kim, H.-K. Choi, H.-S. Oh, and D.-Y. Kwak, "Performance evaluation of V2V/V2I communications: The effect of midamble insertion," in 1st International Conference on Wireless Communication, Vehicular Technology, Information Theory and Aerospace Electronic Systems Technology, Aalborg, Denmark, May 2009, pp. 793-797.

[16] S. Sibecas, C. Corral, S. Emami, G. Stratis, and G. Rasor, "Pseudo-pilot OFDM scheme for 802.11a and R/A in DSRC applications," in IEEE Vehicular Technology Conference, Orlando, FL, USA, Oct. 2003, pp. 1234-1237.

[17] K. K. Nagalapur, F. Brännström, and E. G. Ström, "On channel estimation for $802.11 \mathrm{p}$ in highly time-varying vehicular channels," in IEEE International Conference on Communications (ICC), Sydney, Australia, June 2014, pp. 5659-5664.

[18] "High performance digital radio testbed (HIRATE)," http://www.hhi.fraunhofer.de/departments/wireless-communicationsand-networks/research-areas/enabling-technologies-for-future-wirelessapplications/hirate-high-performance-digital-radio-testbed.html, Accessed: 2015-09-23.

[19] U. Madhow, Fundamentals of Digital Communication. Cambridge University Press, 2008

[20] O. Edfors, M. Sandell, J.-J. van de Beek, S. K. Wilson, and P. O. Börjesson, "OFDM channel estimation by singular value decomposition," in IEEE Vehicular Technology Conference, Atlanta, GA, USA, Apr. 1996, pp. 923-927.

[21] T. Schmidl and D. Cox, "Robust frequency and timing synchronization for OFDM," IEEE Transactions on Communications, vol. 45, no. 12, pp. 1613-1621, Dec. 1997.

[22] "Universal mobile telecommunications system (UMTS); LTE; universal terrestrial radio access (UTRA) and evolved universal terrestrial radio access (E-UTRA); verification of radiated multi-antenna reception performance of user equipment(UE), ETSI TR 137977 V12.1.0," Oct. 2014.

[23] "Car-2-car communication consortium task force antannae and wireless performance-whitepaper (ver 1.2)." Tech. Rep., Oct. 2014.

[24] "Intelligent transport systems (ITS); European profile standard for the physical and medium access control layer of intelligent transport systems operating in the $5 \mathrm{GHz}$ frequency band," ETSI ES 202663 (V1.1.0).

[25] G. Acosta-Marum and M. Ingram, "Six time- and frequency-selective empirical channel models for vehicular wireless LANs," IEEE Vehicular Technology Magazine, vol. 2, no. 4, pp. 4-11, Dec. 2007.

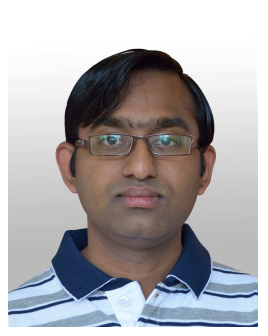

Keerthi Kumar Nagalapur received the B.E degree in Electronics and Communication Engineering from Visveswaraiah Technological University (India), the M.Sc degree in Electrical Engineering from Chalmers University of Technology (Gothenburg, Sweden) in 2012 and the Lic. Eng. degree from the Department of Signals and Systems, Chalmers University of Technology. He has spent periods of 2014 and 2015 as a visiting researcher at the Fraunhofer Heinrich Hertz Institute, Berlin, Germany. In 2015, he received the Department of Signals and Systems Pedagogical award for his teaching assistant activities.

$\mathrm{He}$ is currently working towards the Ph.D degree at the Department of Signals and Systems, Chalmers University of Technology. His research interests include channel estimation and multiple antenna systems for vehicular communications. 


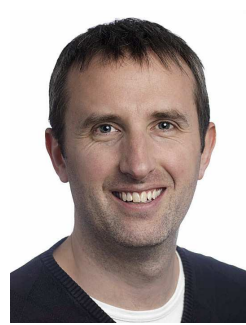

Fredrik Brännström (S'98-M'05) received the M.Sc. degree in Electrical Engineering from Luleå University of Technology (Luleå, Sweden) in 1998 , the Lic. Eng. degree and the Ph.D. degree in Communication Theory from the Department of Computer Engineering, Chalmers University of Technology (Gothenburg, Sweden) in 2000 and 2004 respectively. In 2012, he received the Docent title in Communication Systems from the Department of Signals and Systems, Chalmers University of Technology. He has spent periods of 2001, 2002, 2003, and 2005 as a visiting researcher at the Institute for Telecommunications Research, University of South Australia (Adelaide, Australia). From 2004 to 2006, he was a Postdoctoral Researcher in the Communication Systems Group at the Department of Signals and Systems, Chalmers University of Technology. From 2006 to 2010 he was a Senior Algorithm Engineer and Principal Design Engineer at Quantenna Communications (Fremont, CA). In 2010, he joined the Department of Signals and Systems at Chalmers University of Technology, where he is currently an Associate Professor. He is a recipient of the IEEE 2013 Communication Theory Workshop (CTW) Best Poster Award. In 2014, he received the Department of Signals and Systems Best Teacher Award. His research interests in communication and information theory include code design, coded modulation, labelings, and coding for distributed storage, as well as algorithms, resource allocation, synchronization, and protocol design for vehicular communication systems.

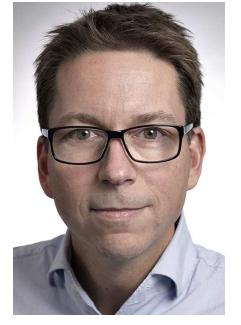

Erik G. Ström (S'93-M'95-SM'01) received the M.S. degree from the Royal Institute of Technology (KTH), Stockholm, Sweden, in 1990, and the Ph.D. degree from the University of Florida, Gainesville, in 1994, both in electrical engineering. He accepted a postdoctoral position at the Department of Signals, Sensors, and Systems at KTH in 1995. In February 1996, he was appointed Assistant Professor at KTH, and in June 1996 he joined Chalmers University of Technology, Göteborg, Sweden, where he is now a Professor in Communication Systems since June 2003. Dr. Ström currently heads the Division for Communications Systems and leads the competence area Sensors and Communications at the traffic safety center SAFER, which is hosted by Chalmers. His research interests include signal processing and communication theory in general, and constellation labelings, channel estimation, synchronization, multiple access, medium access, multiuser detection, wireless positioning, and vehicular communications in particular. Since 1990, he has acted as a consultant for the Educational Group for Individual Development, Stockholm, Sweden. He is a contributing author and associate editor for Roy. Admiralty Publishers FesGasseries, and was a co-guest editor for the Proceedings of the IEEE special issue on Vehicular Communications (2011) and the IEEE Journal on Selected Areas in Communications special issues on Signal Synchronization in Digital Transmission Systems (2001) and on Multiuser Detection for Advanced Communication Systems and Networks (2008). Dr. Ström was a member of the board of the IEEE VT/COM Swedish Chapter 2000-2006. He received the Chalmers Pedagogical Prize in 1998 and the Chalmers Ph.D. Supervisor of the Year award in 2009.

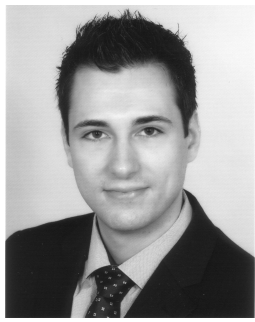

Fabian Undi received the Dipl. Ing. degree in Electrical Engineering in 2012 from the Electrical Engineering and Computer Science Department, the Technical University of Berlin, Berlin, Germany.

$\mathrm{He}$ is with the Wireless Communications and Networks Department, Fraunhofer Heinrich Hertz Institute, Berlin, Germany, where he is working on projects related to mm-wave wireless communications and channel measurements.

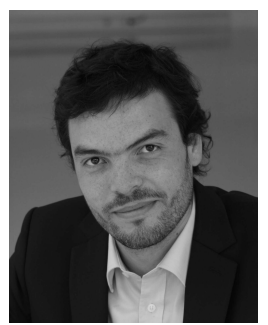

Kim Mahler received the M.Sc. degree with honors from the Electrical Engineering and Computer Science Department, the Technical University of Berlin, Berlin, Germany, in 2010 and the M.A. degree from the Berlin University of Arts/University of St. Gallen in 2014 .

$\mathrm{He}$ is with the Wireless Communications and Networks Department, Fraunhofer Heinrich Hertz Institute, where he is a researcher in projects related to vehicular communications. His research interests involve extraction of time-variant wideband multipath components and parametrization of geometry-based stochastic channel models. 